\title{
Assessment of Landsat 7 Scan Line Corrector-off data gap-filling methods for seagrass distribution mapping
}

\begin{abstract}
Methods to predict and fill Landsat 7 Scan Line Corrector (SLC)-off data gaps are diverse and their usability is case specific. An appropriate gap-filling method that can be used for seagrass mapping applications has not been proposed previously. This study compared gapfilling methods for filling SLC-off data gaps with images acquired from different dates at similar mean sea-level tide heights, covering the Sungai Pulai estuary area inhabited by seagrass meadows in southern Peninsular Malaysia. To assess the geometric and radiometric fidelity of the recovered pixels, three potential gap-filling methods were examined: (a) geostatistical neighbourhood similar pixel interpolator (GNSPI); (b) weighted linear regression (WLR) algorithm integrated with the Laplacian prior regularization method; and (c) the local linear histogram matching method. These three methods were applied to simulated and original SLC-off images. Statistical measures for the recovered images showed that GNSPI can predict data gaps over the seagrass, non-seagrass/water body, and mudflat site classes with greater accuracy than the other two methods. For optimal performance of the GNSPI algorithm, cloud and shadow in the primary and auxiliary images had to be removed by cloud removal methods prior to filling data gaps. The gap-filled imagery assessed in this study produced reliable seagrass distribution maps and should help with the detection of spatiotemporal changes of seagrasses from multi-temporal Landsat imagery. The proposed gap-filling method can thus improve the usefulness of Landsat 7 ETM+ SLC-off images in seagrass applications.
\end{abstract}

